\title{
Classification locale et utilisation des sols en zone sahélienne au Burkina Faso
}

\author{
Roger KISSOU ${ }^{1 *}$, Zacharia GNANKAMBARY ${ }^{2}$, Hassane Bismarck NACRO ${ }^{3}$ et \\ Michel Papoaba SEDOGO ${ }^{2}$
}

\author{
${ }^{1}$ Bureau National des Sols (BUNASOLS) 03 BP 7142 Ouagadougou 03 Burkina Faso. \\ ${ }^{2}$ Institut de l'Environnement et de Recherches Agricoles (INERA) 04 BP 8645 Ouagadougou 04 Burkina Faso. \\ ${ }^{3}$ Université Nazi Boni, Laboratoire d'Etude et de Recherche sur la Fertilité du Sol (LERF) \\ 01 BP 1091 Bo bo-Dioulasso 01, Burkina Faso. \\ *Auteur correspondant ; E-mail : kissouroger@yahoo.fr
}

\section{RESUME}

Le sahel burkinabé est caractérisé par une faible pluviosité et de fortes températures qui ont contraint les paysans à s'adapter à l'adversité du climat. L'objectif de l'étude est d'investiguer sur les systèmes de classification et d'utilisation des sols en milieu peulh. Les travaux ont été menés à Soum-Bella au Burkina Faso à partir de deux toposéquences choisies en fonction de la géologie et de la géomorphologie. La méthode d'entretien collectif a été administrée à 30 paysans peulh en vue d'appréhender les sols majeurs et leur utilisation. Les sols ont été décrits et classifiés sur le terrain par trois personnes ressources. Les résultats ont montré que les critères de classification sont basés sur la topographie et la morphologie des sols qui sont inégalement utilisés pour des raisons socio-culturelles. La perception des paysans peulh des propriétés des sols s'est avérée conforme aux résultats des analyses de laboratoire, confirmant la bonne connaissance des sols par les paysans peulh et la pertinence de leurs critères de classification.

(C) 2018 International Formulae Group. All rights reserved.

Mots clés : Classification locale, utilisation des sols, milieu peulh, Soum-Bella, province du Soum, Burkina Faso.

\section{Local soil classification and land use in sahelian zone in Burkina Faso} \begin{abstract}
In Burkina Faso, the Sahel is characterized by low rainfall and high temperatures that constraint farmers to adapt to adverse climatic conditions. The objective of the study is to investigate soil classification and land use systems in Fulani zone of Burkina Faso. The study was carried out in Soum-Bella village, located in the Soum province. Two topo sequences were selected according to geology and geomorphology. The group interview was administrated to 30 Fulani farmers to apprehending the major soils type and their use. Soils have been described and classified in the field by three resource persons. The results showed that the classification criteria are based on topography and soil morphology that are differently used for socio-cultural reasons. The Fulani farmers' perceptions of soil properties were consistent with the results of the laboratory analyzes, confirming their good knowledge of the soils and the relevance of their classification criteria.

(C) 2018 International Formulae Group. All rights reserved.
\end{abstract}

Keywords: Local classification, land use, Fulani, Soum Province, Burkina Faso. 


\section{INTRODUCTION}

Les paysans sahéliens de l'Afrique de l'Ouest sont confrontés à la récurrence des sécheresses, à la forte dessiccation et à la dégradation des sols (Osbahr et Allan, 2003 ; Da et al., 2008). Dans la zone soudanosahélienne, et particulièrement dans le sahel burkinabé, les sols ferrugineux tropicaux qui sont dominants, sont caractérisés par une déficience en colloïdes organo-minéraux avec une activité physico-chimique de surface faible (Benoit, 2008). Les températures élevées et les conditions humides en saison pluvieuse, ont accéléré la minéralisation de la matière organique de sorte que le taux est particulièrement bas $(0,5 \%)$ dans cette zone semi-aride où la production de biomasse végétale est limitée et où les sols sont le plus souvent sableux (Pallo et al., 2009 ; Ouandaogo et al., 2016).

Face à l'adversité du climat, les paysans ont utilisé leurs connaissances locales pour subvenir à leurs besoins socioéconomiques (Yaméogo, 2010). Malgré leur ignorance de la formation et de l'évolution des sols, les paysans parviennent à percevoir les potentialités et les contraintes des sols de leur terroir (Birmingham, 2003 ; Gray et Morant, 2003 ; Zerbo et al., 2016). Ces connaissances les orientent dans la prise de décision, particulièrement dans le choix des modes d'utilisation des sols (Niemeijer et Mazzucato, 2003). Elles peuvent être sources de développement des technologies pour les pays au sud du Sahara et particulièrement pour le Burkina Faso (Gray et Morant, 2003 ; Kissou et al., 2014).

Cependant, les informations relatives à la classification locale et à l'utilisation des sols en zone sahélienne, au Burkina Faso, sont quasi-rares. Aussi, l'objectif de ce travail est d'appréhender les systèmes de classification locale et les types d'utilisation des sols en milieu peulh, à partir du village de SoumBella situé à l'extrême Nord de la province du Soum, en zone sahélienne, au Burkina Faso.

\section{MATERIEL ET METHODES Milieu biophysique}

L'étude a été menée en zone sahélienne du Burkina Faso, dans l'extrême
Nord de la province du Soum où est situé le village de Soum-Bella ( $1^{\circ} 07^{\prime} 09^{\prime}$ 'W, $14^{\circ} 44^{\prime}$ 53 '’ N).

Le climat est de type sahélien. La pluviosité moyenne annuelle enregistrée dans les stations synoptiques proches du site d'étude varie de $409 \mathrm{~mm}$ à $453 \mathrm{~mm}$. La température moyenne annuelle est de $29,8{ }^{\circ} \mathrm{C}$. Le substratum géologique est constitué, des formations cristallines anciennes $\mathrm{du}$ précambrien inférieur recouvertes en discordance par des matériaux sédimentaires (Donzeau et al., 2003). La géomorphologie est caractérisée par des cordons dunaires d'origine éolienne qui correspondent aux ergs anciens et récents. La végétation est de type steppique, caractérisée par une strate herbacée discontinue, courte, maigre et une strate arborée et arbustive très clairsemée (Guinko, 1998). La couverture pédologique selon la CPCS (1967), est formée de vertisols, de sols bruns sub-arides et de sols hydromorphes (Kissou, 2002). Les activités humaines sont largement dominées par l'élevage ; l'agriculture occupe une place secondaire. Le système de culture est à base de céréales avec culture continue du mil qui occupe $96 \%$ de la superficie des céréales; et constitue $91 \%$ de la production céréalière (DPSAA, 2013) (Direction de la Prospective et des Statistiques Agricoles et Alimentaires). La langue la plus parlée est le fulfuldé.

\section{Méthodes}

La méthode d'enquête utilisée a été l'entretien collectif, administré à un groupe de 30 paysans peulh en vue d'appréhender la classification locale et les types d'utilisation des terres pratiqués. Trois personnes ressources ont été ensuite désignées par le chef de village pour décrire et classifier les sols en langue peulh. Les sols ont été décrits sur deux toposéquences choisies sur la base de la géologie et de la géomorphologie. La méthode de description utilisée par les paysans peulh a été la méthode visuelle pour caractériser la couleur du sol, la topographie, les états de surface et la végétation. La texture du sol a été appréciée par la méthode tactile. 
La taxonomie locale a été faite sur la base de l'interprétation des propriétés des différents caractères observés dans l'épipédon.

Pour établir une corrélation avec la classification scientifique, des fosses pédologiques ont été ouvertes sur les mêmes toposéquences et aux mêmes points où l'épipédon a été décrit par les paysans peulh. Les fosses pédologiques ont été décrites selon les Directives FAO (1994). Les sols ont été classifiés selon la CPCS (1967) et la WRB (2006).

Des prélèvements d'échantillons ont été effectués à la profondeur de $0-20 \mathrm{~cm}$. Les échantillons ont été analysés au laboratoire du Bureau National des Sols (BUNASOLS) en vue de déterminer la texture (Bouyoucos hydrometer, 1927), les constantes hydriques $\mathrm{pF} 2,5$ et $\mathrm{pF} 4,2$ (plaques céramiques), les teneurs en carbone total (Walkley et Black, 1934), l'azote total (Kjeldahl Hhillebrand, 1953), le phosphore assimilable (Bray et Kurtz, 1945), le potassium disponible (FAO, 1984), les bases échangeables et la capacité d'échange cationique (méthode à l'argent thiouré) et le $\mathrm{pH}_{\text {eau }}$ (méthode électrométrique).

La transcription des noms locaux des sols en langue fulfuldé a été assurée par les formateurs $\mathrm{du}$ centre d'alphabétisation $\mathrm{du}$ village de Sibé $\left(1^{\circ} 30^{\prime} 36^{\prime}\right.$ ' W, $14^{\circ} 06^{\prime} 30^{\prime}$, $\mathrm{N})$ et confirmée par l'Institut National de l'Alphabétisation (INA) du Burkina Faso.

Les résultats ont été validés à Djibo, chef-lieu de la province du Soum, lors d'un atelier de restitution par une assemblée composée des chefs coutumiers, des représentants des associations et groupements villageois des différentes localités et des structures administratives et de recherche qui travaillent dans la province. La Figure 1 présente la zone d'étude.

\section{Analyse statistique}

Les données quantitatives des propriétés physico-chimiques ont été comparées par une analyse de variance (ANOVA) avec le logiciel XLSTAT.

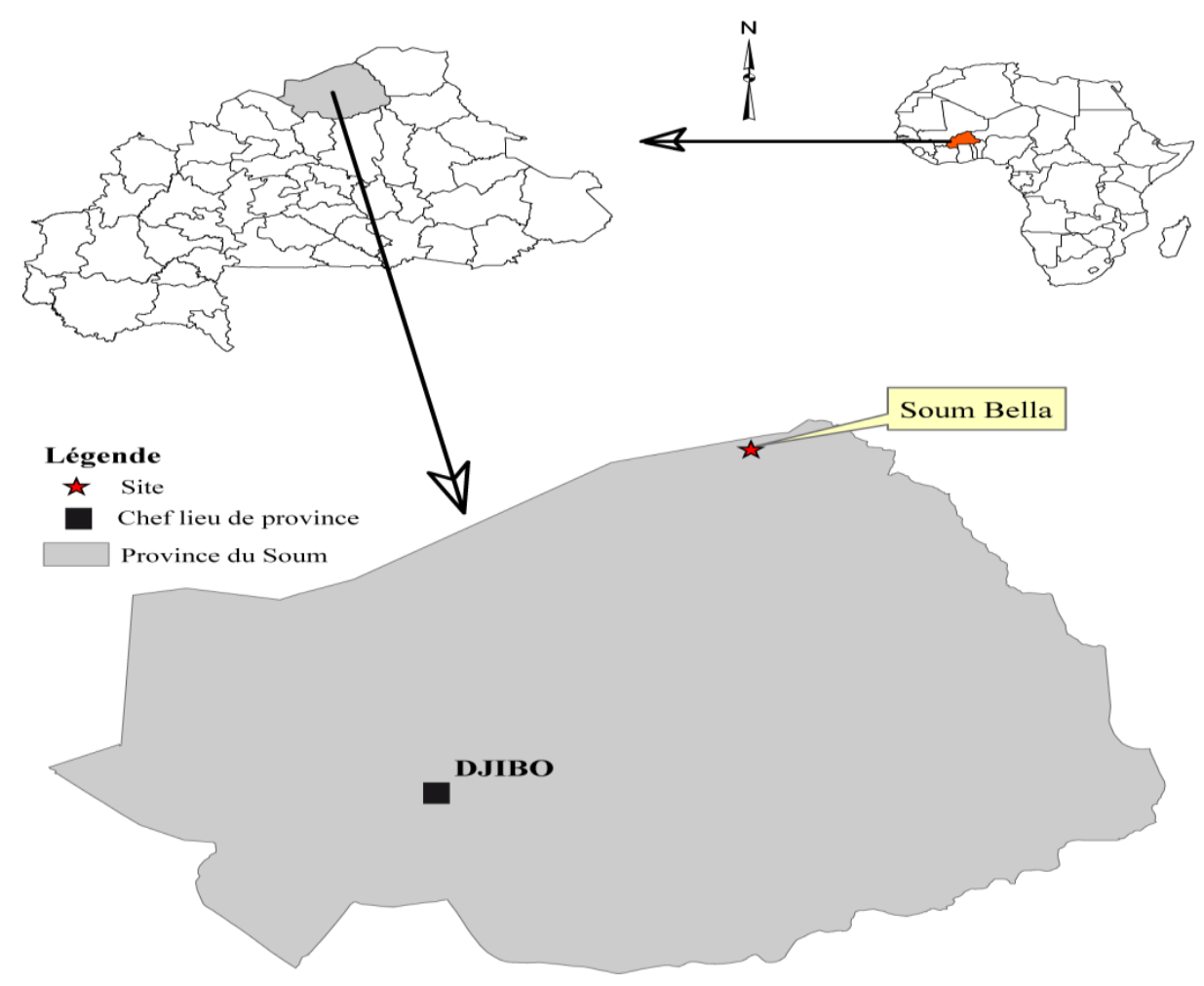

Figure 1: Localisation du site étudié. 


\section{RESULTATS}

\section{Classification locale des sols}

La différenciation des types de sols a été faite par les peulh du village de SoumBella, selon deux critères déterminants : les critères topographiques et morphologiques.

\section{Critères topographiques}

Les paysans peulh ont cité comme composantes de la topographie, le seeno et le weendu.

Le terme seeno a été utilisé pour désigner le cordon dunaire qui longe la mare de Soum. Les seeno ont été qualifiés de sols sableux, perméables avec une faible rétention en eau. La couleur de surface relevée à l'aide du Code Munsell (2000), est jaune rougeâtre (7,5 YR6/6). Les seeno correspondent aux sols bruns rouges sub-arides (CPCS, 1967) et aux arénosols rubiques-protiques (aridiques) (WRB, 2006).

Les paysans peulh ont attribué le nom weendu aux sols de la plaine alluviale de la mare de Soum. Les weendu ont été décrits comme étant des sols mal drainés, ayant une bonne rétention en eau. Selon la classification scientifique, ils appartiennent au groupe de référence des gleysols de la WRB (2006) et à la classe des sols hydromorphes de la CPCS (1967).

\section{Critères morphologiques}

Le principal critère morphologique estimé essentiel par les paysans peulh dans la caractérisation des sols a été la texture. Les sols très collants, qui adhèrent fortement aux doigts et s'étirent lorsqu'on écarte les doigts, ont été appelés bolaawo par les paysans. Les bolaawo sont formées des alluvions argileuses qui occupent la quasi-totalité de la partie inondable de la mare de Soum. De l'avis des paysans peulh, ils sont peu perméables et sont dotés d'une bonne rétention en eau. Les bolaawo correspondent aux vertisols et aux sols bruns sub-arides vertiques selon la CPCS (1967), aux vertisols et cambisols d'après la WRB (2006).

Le Tableau 1 présente une synthèse de la classification peulh et les correspondances avec la CPCS (1967) et la WRB (2006).

La Figure 2 présente la toposéquence type des sols de Soum-Bella en milieu sédimentaire.
Les résultats de l'analyse des propriétés physico-chimiques des sols sont présentés dans le Tableau 2. Celles-ci sont différentes selon les critères topographiques et morphologiques. Les seeno ont un taux de sables très élevé $(75,5 \%)$, une très faible teneur en argile $(4,9 \%)$ et une faible réserve utile en eau $(18,7 \mathrm{~mm})$. Tous les paramètres de fertilité sont caractérisés par de faibles valeurs à l'exception du potassium disponible $\left(77,4 \mathrm{mg} . \mathrm{kg}^{-1}\right)$. La fraction fine (argile + limons) est dominante dans bolaawo $(79 \%)$ et weendu $(75 \%)$ qui présentent des réserves utiles en eau élevées dans les 50 premiers centimètres de sol, respectivement 120 et 133 $\mathrm{mm}$. Les bolaawo enregistrent les plus grandes valeurs pour la somme des bases échangeables ( $14 \mathrm{cmol}^{\mathrm{kg}} \mathrm{kg}^{-1}$ bolaawo $_{1}$ et 15,4 cmol. $\mathrm{kg}^{-1}$ bolaawo $)$ et la capacité d'échange cationique (17 cmol. $\mathrm{kg}^{-1}$ bolaawo $_{1}$ et 19 cmol. $\mathrm{kg}^{-1}$ bolaawo $_{2}$ ). Les teneurs en carbone et en azote sont plutôt élevées dans le weendu (respectivement, 9,1 mg. $\mathrm{g}^{-1}$ et $0,7 \mathrm{mg} \cdot \mathrm{g}^{-1}$ ) que dans les autres sols. Tous les sols se sont singularisés par leur extrême déficience en phosphore.

\section{Utilisation des terres}

Les types de sols décrits par les paysans peulh sont inégalement utilisés. De leur avis, les seeno sont les plus cultivés car ils sont meubles, faciles à travailler et ont une bonne aptitude au mil, au niébé et au calebassier. Ils sont aussi adaptés aux outils aratoires traditionnels. Cependant, les paysans peulh ont affirmé que la fertilité des seeno est plus basse que celle des bolaawo et des weendu.

De l'avis des paysans peulh, les bolaawo sont fertiles mais sont lourds, très collants à l'état humide et très durs à l'état sec. Malgré leurs propriétés physicochimiques favorables à l'agriculture, ils ne sont pas exploités par les paysans peulh. En saison de pluie, les bolaawo sont considérés comme des sols où les risques d'embourbement du gros bétail sont très élevés. 
Tableau 1: Correspondance entre les classifications Peulh, CPCS (1967) et WRB (2006).

\begin{tabular}{lcc}
\hline Classification Peulh & Classification CPCS (1967) & Word reference base (2006) \\
\hline $\begin{array}{l}\text { Seeno } \\
\text { Weendu }\end{array}$ & Sol brun rouge sub-aride & Arénosol rubique-protique (aridique) \\
& $\begin{array}{c}\text { Sol hydromorphe peu humifère à } \\
\text { redistribution du calcaire à nodules }\end{array}$ & Gleysol calcique (eutrique) \\
Bolaawo $_{1}$ & Sol brun-subaride vertique & Cambisol vertique (eutrique) \\
Bolaawo $_{2}$ & Vertisol vertique & Vertisol mazique (eutrique) \\
\hline
\end{tabular}

Environnement du profil
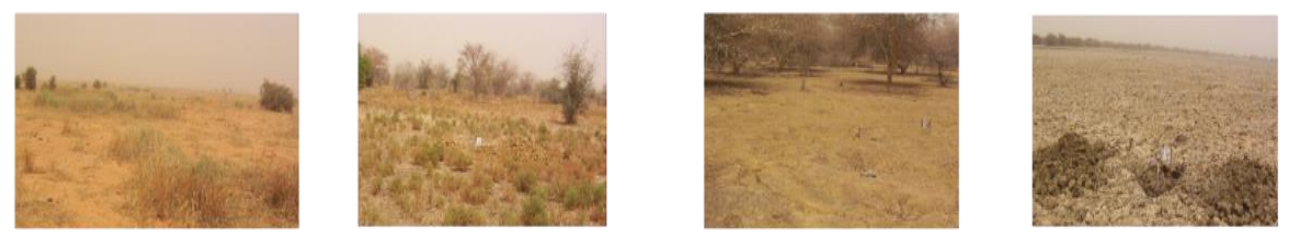

Toposéquence

Profils

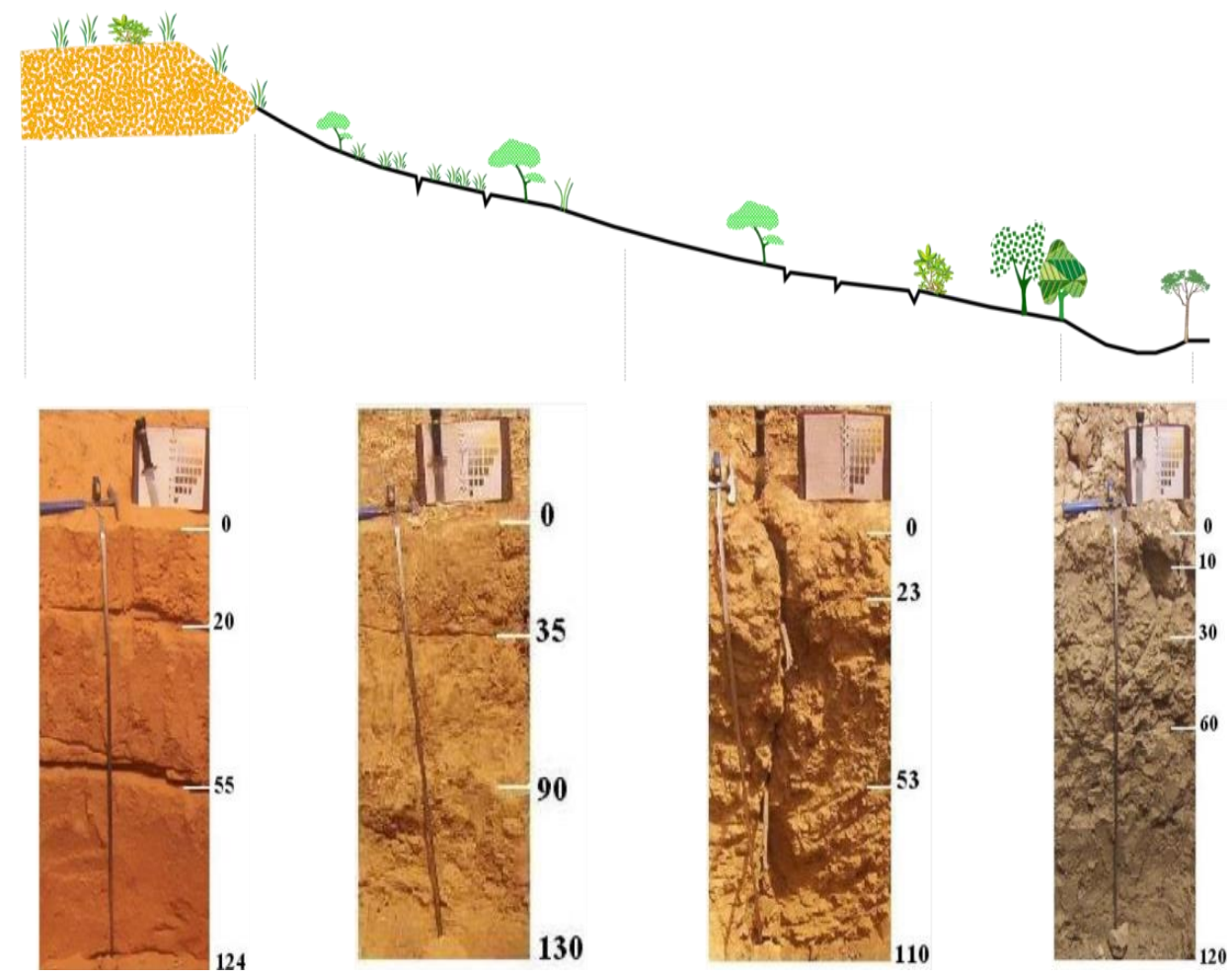

Classification en fulfuldé

Classification WRB (2006)

Géomorphologie

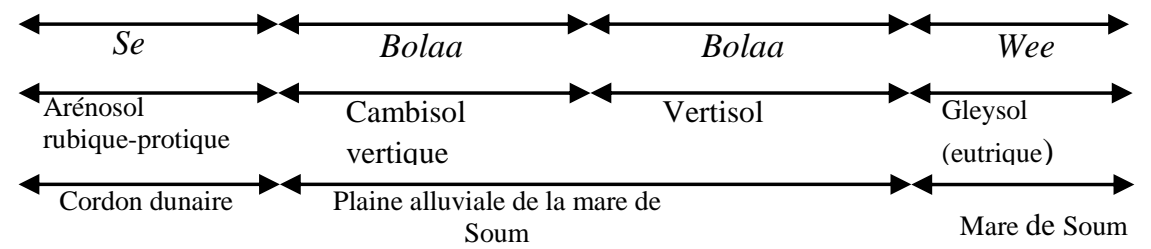

Figure 2 : Toposéquence type des sols de Soum-Bella en milieu sédimentaire. 
Tableau 2 : Caractéristiques physico-chimiques des sols.

\begin{tabular}{|c|c|c|c|c|c|c|}
\hline \multirow[t]{2}{*}{ Paramètres } & \multicolumn{6}{|c|}{ Types de sols } \\
\hline & Seeno & Bolaawo $_{1}$ & Bolaawo $_{2}$ & Weendu & Test SNK & Probabilité \\
\hline Argile \% & $4,9^{\mathrm{c}}$ & $26,2^{\mathrm{b}}$ & $53,3^{\mathrm{a}}$ & $45,8^{\mathrm{a}}$ & HS & $<0,0001$ \\
\hline Limons \% & $20,6^{\mathrm{a}}$ & $31,7^{\mathrm{a}}$ & $25,5^{\mathrm{a}}$ & $29,4^{\mathrm{a}}$ & NS & $<0,1$ \\
\hline Sables \% & $75,5^{\mathrm{a}}$ & $42,2^{\mathrm{b}}$ & $21,1^{\mathrm{c}}$ & $17,4^{\mathrm{c}}$ & HS & $<0,0001$ \\
\hline $\mathrm{RU}(\mathrm{mm})$ & $18,7^{\mathrm{c}}$ & $87,3^{\mathrm{b}}$ & $120,2^{\mathrm{a}}$ & $133,2^{\mathrm{a}}$ & HS & $<0,0001$ \\
\hline $\mathrm{C}\left(\mathrm{mg} \cdot \mathrm{g}^{-1}\right)$ & $2,1^{\mathrm{b}}$ & $4,0^{\mathrm{a}}$ & $5,4^{\mathrm{a}}$ & $9,1^{\mathrm{a}}$ & NS & $<0,1$ \\
\hline $\mathrm{N}\left(\mathrm{mg} \cdot \mathrm{g}^{-1}\right)$ & $0,2^{\mathrm{c}}$ & $0,3^{\mathrm{c}}$ & $0,5^{\mathrm{b}}$ & $0,7^{\mathrm{a}}$ & HS & $<0,004$ \\
\hline $\mathrm{Kd}\left(\mathrm{mg} \cdot \mathrm{kg}^{-1}\right)$ & $77,4^{\mathrm{a}}$ & $114^{\mathrm{a}}$ & $252,3^{\mathrm{a}}$ & $120^{\mathrm{a}}$ & NS & $<0,1$ \\
\hline $\mathrm{Pa}\left(\mathrm{mg} . \mathrm{kg}^{-1}\right)$ & $3,0^{\mathrm{a}}$ & $1,5^{\mathrm{a}}$ & $1,5^{\mathrm{a}}$ & $1,4^{\mathrm{a}}$ & NS & $<0,1$ \\
\hline $\mathrm{S}\left(\mathrm{cmol} . \mathrm{kg}^{-1}\right)$ & $1,0^{\mathrm{b}}$ & $14,0^{\mathrm{a}}$ & $15,4^{\mathrm{a}}$ & $9,1^{\mathrm{a}}$ & HS & $<0,0001$ \\
\hline $\mathrm{CEC}\left(\mathrm{cmol} . \mathrm{kg}^{-1}\right)$ & $1,5^{\mathrm{b}}$ & $16,9^{\mathrm{a}}$ & $19,0^{\mathrm{a}}$ & $12,8^{\mathrm{a}}$ & HS & $<0,0001$ \\
\hline $\mathrm{pH}$ & $6,4^{\mathrm{a}}$ & $6,6^{\mathrm{a}}$ & $6,7^{\mathrm{a}}$ & $5,7^{\mathrm{a}}$ & NS & $<0,0001$ \\
\hline
\end{tabular}

Les weendu sont surtout utilisés par les paysans peulh comme des lieux d'abreuvement du bétail et les abords comme aire de repos des animaux particulièrement à la sortie de la saison des pluies. Toutefois, de petits périmètres potagers entretenus par les femmes sont parfois observés. De manière générale, sur le plan agricole, les weendu sont très peu exploités par les paysans peulh.

\section{DISCUSSION}

Les paysans peulh du sahel burkinabé ont une bonne connaissance des sols de leur terroir qu'ils exploitent sur la base de critères topographiques et morphologiques pertinents et de l'expérience. Des études précédentes (Birmingham, 2003 ; Gray et Morant, 2003 ; Niemeijer et Mazzucato, 2003 ; WinklerPrints, 2003; Dialla, 2004) ont également fait cas de la bonne connaissance des sols par les paysans. Il est très souvent à tort de considérer que le peulh n'a pas d'attache à la terre. L'espace rural peulh est structuré selon les types d'utilisation des sols à l'image des autres terroirs du Burkina Faso (Aquino, 2000; Niemeijer et Mazzucato, 2003). Cependant, les sols sont inégalement utilisés. L'avis des paysans peulh sur les propriétés des seeno, des bolaawo et des weendu a été confirmé par les résultats physico-chimiques des sols. Les bolaawo et des weendu ont effectivement une fertilité naturelle élevée tandis que les seeno sont caractérisés par un faible niveau de fertilité. Malgré leurs bonnes qualités avérées, les bolaawo et les weendu ne sont pas cultivés par les paysans peulh pour des raisons socioculturelles. Les cultures qui sont adaptées à ces milieux (maïs, sorgho et riz) n'entrent pas dans leurs habitudes alimentaires. Ce sont également des sols lourds, inadaptés à l'hilaire, principal outil aratoire des paysans peulh.

Sur le plan granulométrique, les seeno ont un taux de sables élevé (75\%); ce qui justifie leur appellation de sols sableux par les paysans peulh. Ce sont des aires privilégiées pour la culture du mil, céréale la plus cultivée qui occupe $96 \%$ de la superficie des céréales et représente $91 \%$ de la production en céréales (DPSAA, 2013) (Direction de la Prospective et des Statistiques Agricoles et Alimentaires). C'est le principal aliment de 
base des paysans peulh, très utilisé dans la préparation de plusieurs mets.

L'une des caractéristiques majeures des sols qui ne peut pas être perçues par les paysans peulh est la pauvreté en phosphore. Les résultats obtenus sont conformes aux études effectuées sur les sols du Burkina, considérés excessivement pauvres en phosphore (Lompo et al., 2008 ; Lompo et al., 2009).

La classification paysanne des sols est locale, bien circonscrite au terroir et spécifique pour des conditions de production locale. Plusieurs auteurs l'ont bien soulignée (Birmingham, 2003 ; Gray et Morant, 2003). Ainsi, au sein d'un même groupe ethnique, le nom d'un type de sol donné peut varier d'un dialecte à l'autre. Pourtant, ces divergences de dénomination ne sont pas uniquement spécifiques à la classification locale. Des disparités existent aussi dans les systèmes de classification nationaux et scientifiques.

\section{Conclusion}

Les paysans peulh du sahel burkinabè ont une bonne connaissance des sols de leurs terroirs. Ces connaissances qui sont spécifiques et adaptées aux conditions de production les aident à identifier les potentialités et les contraintes de leurs sols et à les utiliser conformément à leurs besoins d'existence. La classification peulh est basée sur des critères topographiques, morphologiques et sur l'expérience. Les champs sont concentrés sur les seeno, sols sableux, meubles, faciles à cultiver avec l'hilaire, bien adaptés au mil, céréale la plus consommée. La perception des paysans peulh des propriétés des sols s'est avérée conforme aux résultats des analyses de laboratoire, confirmant la bonne connaissance des sols par les paysans peulh et la pertinence de leurs critères de classification. Il s'avère maintenant nécessaire de prendre en compte les connaissances locales dans les programmes et projets de recherche pour mieux les comprendre et établir de plus en plus, des corrélations avec les systèmes de classification scientifique. C'est une classification simple et pratique qui peut contribuer efficacement à une cartographie peu coûteuse des sols et à une gestion durable des terres.

\section{CONFLITS D'INTERETS}

Les auteurs, à l'unanimité ont affirmé qu'il n'y avait pas de conflit d'intérêts.

\section{CONTRIBUTION DES AUTEURS}

RK est le principal meneur de l'étude. ZG a contribué au suivi régulier du déroulement de l'étude et à l'analyse statistique des données physico-chimiques des sols. HBN et MPS ont joué un rôle crucial dans la lecture et l'amendement du document.

\section{REFERENCES}

Benoît E. 2008. Les changements climatiques: vulnérabilité, impacts et adaptation dans le monde de la médecine traditionnelle au Burkina Faso. VertigO, 8(1). Doi: 10.4000/vertigo. 14616

Birmingham DM. 2003. Local knowledge of soils: the case of contrast in Côte d'Ivoire. Geoderma, 111: 481-502. Doi: S0016-7061 (02)00278-1

Bouyoucos GJ. 1927. Directions for determining the colloidal materiel of soil by hydrometer method. Science Magazine, 66(1696): 16-17.

Bray RH, Kurtz LT. 1945. Determination of total organic and available forms of phosphorous in soils. Soil Science, 59: 39-45.

Commission de pédologie et de Cartographie des Sols (CPCS). 1967. Classification des Sols. ENSA-GRIGNON : France, 87.

Da DEC, Hamma Y, Yonkeu S. 2008.Unités géomorphologiques et gestion de la fertilité des sols dans le Centre-Nord du Burkina Faso par les populations locales. Int. J. Biol. Chem. Sci., 2(3): 306-315.

Dialla BE. 2004. Les savoirs locaux: un capital culturel souvent occulté. Série Document de Travail, DT-CAPES, Ouagadougou, Burkina Faso ; 32.

Donzeau M, Chevemont P, Metour J, Koté S, Ouédraogo I, Castaing C, Kaboré EB, Billa M, Zida B. 2003. Carte géologique 
du Burkina Faso à l'échelle 1: 200 000, feuille de Djibo.

Direction de la Prospective et des Statistiques Agricoles et Alimentaires (DPSAA). 2013. Résultats définitifs de la campagne agricole et de la situation alimentaire et nutritionnelle 2012-2013, 49.

FAO. 1984. Méthodes d'analyse physique et chimique des sols et des eaux. Bulletin Pédologique de la FAO, 10: Rome, 280.

FAO, ISRIC. 1994. Directives pour la description des sols. ( $3^{\text {ème }}$ édn), Service des sols-ressources, aménagement et conservation, Division de la mise en valeur des terres et des eaux. FAO, ISRIC, 75.

Gray LC, Morant P. 2003. Reconciling indigenous knowledge with scientific assessment of soil fretility changes in southwestern Burkina Faso. Geoderma, 111: 425-437. Doi : S0016-7061 (02) 00275-6

Guinko S. 1998. Caractéristiques de la végétation du Burkina Faso et leurs impacts sur les sols, p. 13

Hillebrand WF, Lundell GE, Bright HA, Hoffman JI. 1953. Applied Inorganic Analysis. John Wiley, Sons, Inc: New York, USA ; 1034.

Kissou R, Traore E, Gnakambary Z, Nacro H B, Sedogo MP. 2014. Connaissance endogène de la classification et de la fertilité des sols en zone SudSoudanienne du Burkina Faso. VertigO, 14 (1). Doi : 10.4000/vertigo. 14616

Kissou R. 2002. Etude morphopédologique de la province du Soum, 55 p. et annexes.

Lompo F, Bonzi M, Bado BV, Ouandaogo N, Sedgo MP, Assa A. 2008. Influence à long terme des modes de gestion de la fertilité sur les états, les formes, les fractions et le bilan du phosphore d'un lixisol du Burkina en culture continue de sorgho. International Journal of Biological and Chemical Sciences, 2(2): 175-184.

Lompo F, Segda Z, Gnankambary Z, Ouandaogo N. 2009. Influence des phosphates naturels sur la qualité et la biodégradation d'un compost de paille de maïs. Tropicultura, 27(2): 105-109.

Munsell. 2000. Soil Color Charts. Munsell Color Company - x-rite.

Niemeijer D, Mazzucato V. 2003. Moving beyond indigenous soils taxonomies: local theories of soils for sustainable development. Geoderma, 111: 403-424. Doi: S0016-7061 (02) 00274-4

Ouandaogo N, Ouattara B, Pouya MB, Gnankambary Z, Naco HB, Sedogo PM. 2016. Effets des fumures organominérales et des rotations culturales sur la qualité des sols. Int. J. Biol. Chem. Sci., 10(2): 904-918. Doi: hpp://dx.doi.org/10. 4314/ijbcs.v10i2.37

Pallo F, Sawadogo N, Zombré N P, Sédogo M P. 2009. Statut de la matière organique des sols de la zone nord-soudanienne au Burkina Faso. Biotechnol. Agro. Soc. Environ., 13(1): 139-142.

Walkley A, Black IA. 1934. An examination method of Detjareff and a proposed modification of the chromic acid titration method. Soil Science, 37: 29-38.

WinklerPrints A M G A. 2003. Local soil knowledge: insights, application and challenges. Geoderma, 111: 165-170. Doi: S0016-7061 (2)00262-8

World Reference Base for soil resources (WRB). 2006. A framework for international classification, correlation and communication. Ed. FAO, World Soil Resources Reports $N^{\circ} 103$, Rome, $128 \mathrm{p}$.

Yaméogo SF. 2010. Rapport de projet sur le programme international de formation sur le changement climatique, atténuation et adaptation, 44p.

Zerbo L, Nacro HB, Yaho-Kouamé A, Sedogo PM. 2016. Connaissances et perceptions locales de la dynamique des cuirasses ferrugineuses: Etude de cas en zone Nord-soudanienne et Sud-soudanienne du Burkina Faso. Int. J. Biol. Chem. Sci., 10(6): 2754-2767. Doi: hpp://dx.doi.org/10. 4314/ijbcs.v10i6.28 